\title{
Structural Investigation of Granular Composites by Modern Methods
}

\author{
I. RÁThy, P. PinKe, E.R. FÁBiÁN, H.E. NAGYNÉ \\ Óbuda University Donát Bánki Faculty of Mechanical and Safety Engineering, Institute of Materials and \\ Manufacturing Science, rathy.istvanne@bgk.uni-obuda.hu
}

\begin{abstract}
Structural investigations of polymer-based particulate composites were carried out using modern test methods. We had composite sheets with different compositions and different injection molding speeds. In the polypropylene matrix, three types of glass beads were mixed in three weight percentages. In our investigations, the granular composites were studied with a widely used examination equipment, scanning electron microscope (SEM) and industrial computer tomography (CT) as a modern diagnostic tools. The purpose of the study was to investigate the distribution and interfacial adhesion of glass beads.
\end{abstract}

Keywords: granular composites, structural investigations, SEM, CT X-ray

\section{Introduction}

Structural investigations of granular composites were carried out using modern test methods. Composite sheets of different compositions and with different injection molding speeds were prepared and used. Polypropylene (PP) matrix was mixed with glass beads of three different size ranges $(0-75 \mu \mathrm{m}, 75-125 \mu \mathrm{m}, 125-250 \mu \mathrm{m})$ and in three weight percentages $(10,25,40 \mathrm{wt} \%)$. During our investigations, scanning electron microscopy (SEM) and industrial computer tomography (CT) were used as modern diagnostic tools.

A scanning electron microscope is an electron-optic device that scans a defined area of the subject's surface with a directional thin electron beam [1]. Signals from the interaction between an electron beam and an object are sensed by suitable detectors and after proper processing and synchronization with the movement of the electron beam, they are visualized [2]. In our case, the amount and size of glass beads were examined at predetermined locations of the obtained sample plates $(80 \times 80 \times 2 \mathrm{~mm})$.

The computer tomographic device generates a 2D X-ray image sequence of the object under examination, in which the sample rotates in series with a defined small angle value [3]. This is repeated until the specimen is completely rotated, and an X-ray image is produced at each angle. Based on 2D images 3D reconstructions can be made with appropriate software [4-6]. 


\section{Investigations with scanning electron microscopy (SEM)}

\subsection{Preparation of test specimens}

Only properly prepared specimens may be used for scanning electron microscopy (SEM). 80x80x2 mm sheets do not fit in the sample holder.

Therefore, first, identifiable specimens of appropriate size had to be cut out. The sheets were cut in half parallel to the injection barrier and then cut perpendicularly at $10 \mathrm{~mm}$ intervals to obtain 4 sample plates from the lower left of the sample plate, serially numbered as shown in Figure 1. The specimens were individually broken with a Charpy impact test equipment. The fracture surface of the specimens was examined. The samples to be tested had to be made conductive. For conductivity the samples were coated with gold steaming.

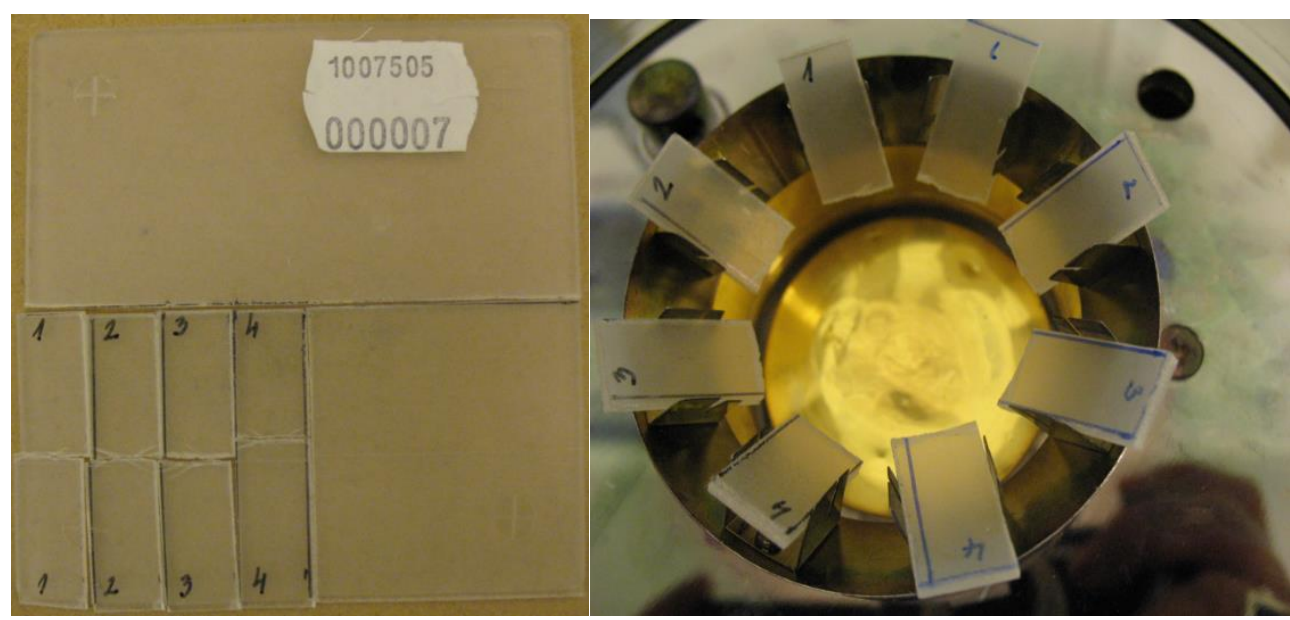

Figure 1. Cut the sheet, and gilding the surface

\subsection{SEM analysis of specimens of the same weight percentage with different bead diameter}

Figure 2 shows SEM images from the fracture surface of a granular composite (at $40 \mathrm{wt} \%$ glass beads content). The images can be used to determine the size and volume fraction of glass beads. The images show that the surface adhesion is good between the matrix and the glass beads, the PP matrix adheres to the glass beads and the fracture occurred in the matrix. The SEM images also show that the distribution of glass beads is not considered to be uniform. When analyzing a large number of SEM images, it is only possible to draw conclusions about the glass bead content and size distribution of the glass beads in a given composite. 


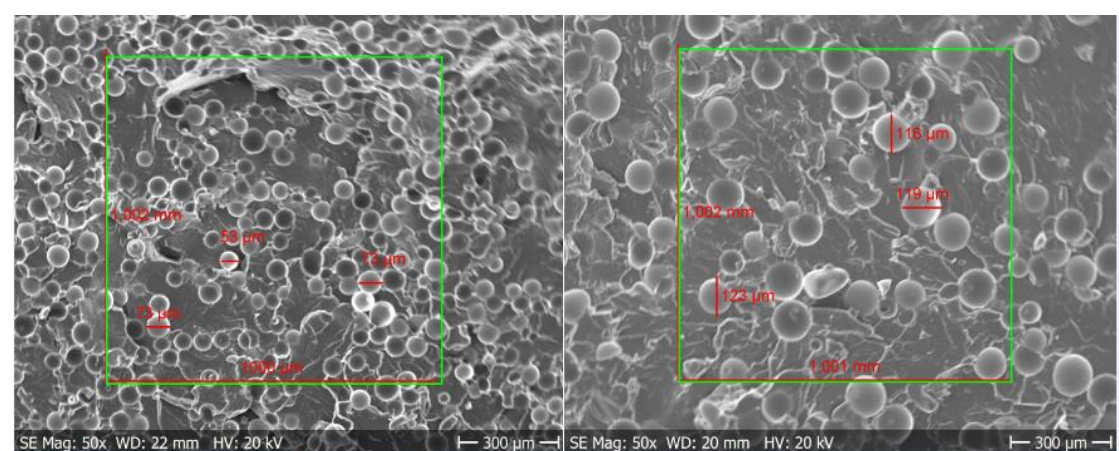

a) 4007520-08-01b sample

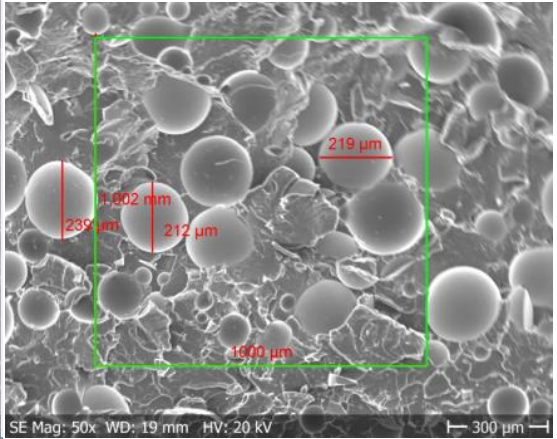

c) 4025020-08-03b sample

Figure 2. SEM images of PP composites containing different bead sizes ( 40 wt \% glass bead content)

\subsection{SEM analysis of specimens of the same bead diameter with different weight percentage}

While the previous composites had the same weight percentage of glass beads, the present composites had the same bead diameters but were mixed in different weight percentages. Figure 3 shows SEM images. Figure 3 a) shows $10 \%$ glass beads content, Figure 3 b) is a $25 \%$ glass bead content, and Figure $3 \mathrm{c}$ ) is a composite fracture surface containing $40 \%$ glass beads. On the fracture surface, the bead diameter can be measured and the number of beads determined.

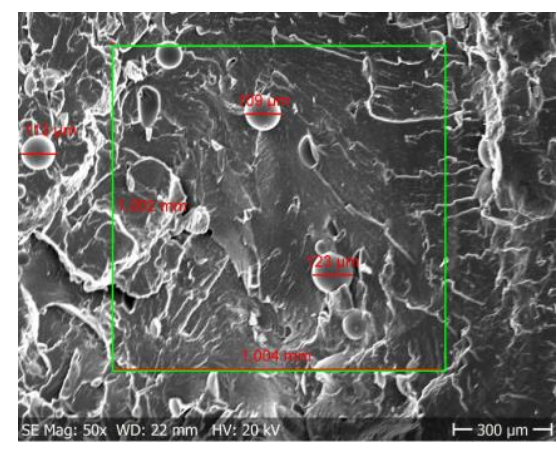

a) $1012505-08-02 a$

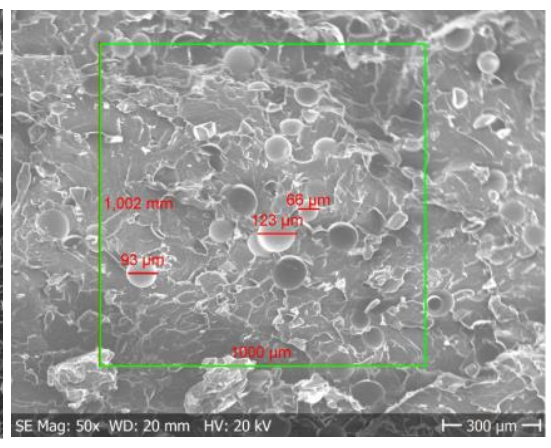

b) $2512505-13-01 \mathrm{~b}$

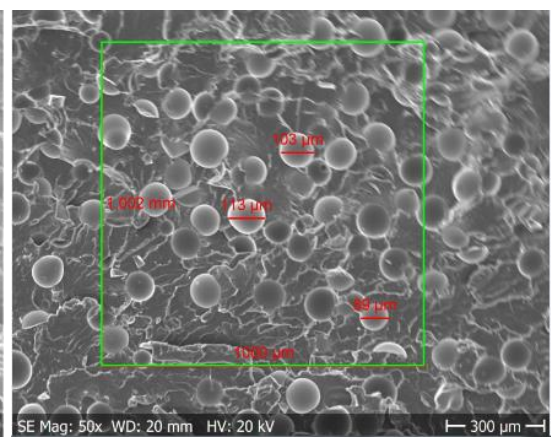

c) $4012505-12-01 \mathrm{~b}$

Figure 3. SEM images of PP composites containing different bead content (same bead diameter)

\section{Investigations with industrial computer tomography (CT)}

\subsection{D CT analysis of specimens of the same bead diameter and same injection molding speeds with different weight percentage}

Figure 4 shows 2D CT images of complete composite sheets (at $10 \mathrm{wt} \%, 25 \mathrm{wt} \%$ and $40 \mathrm{wt} \%$ glass beads content). Composites containing different amounts of glass beads show different injection molding patterns, ranging from homogeneous distribution through "tunnel" shape to "volcanic" shape. The darker regions on the images indicate the density increase of the glass beads. 


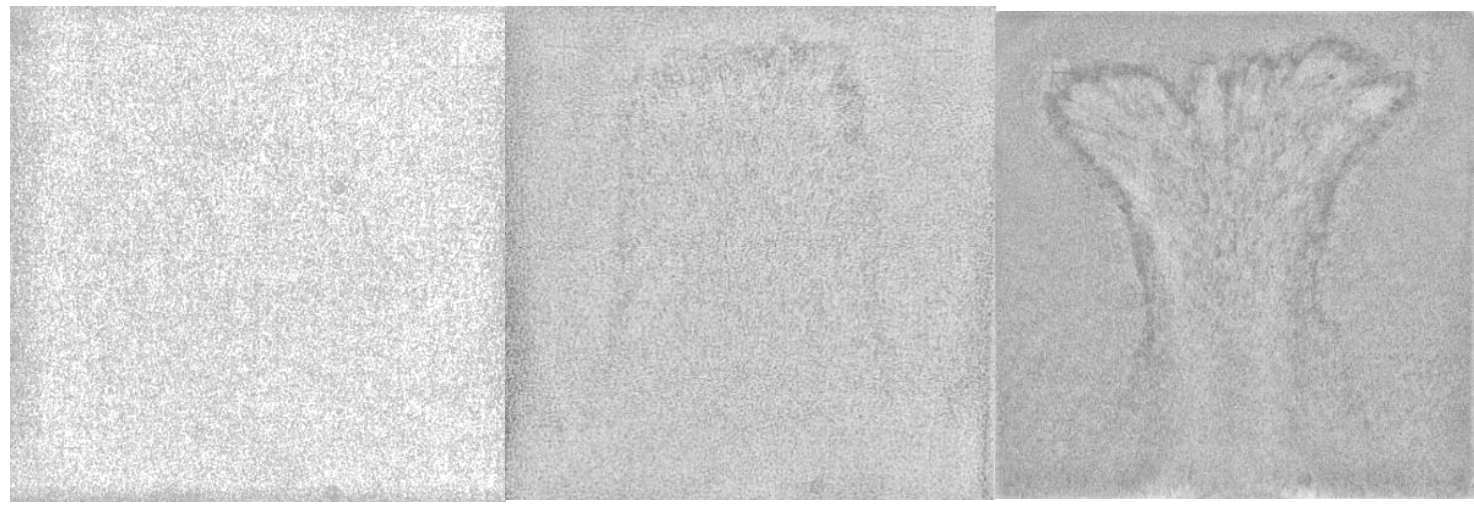

a) 1007505 sample

b) 2507505 sample

c) 4007505 sample

Figure 4. 2D CT images of PP composite samples containing different glass bead content (10, 25, 40 wt \%) produced at the same injection molding speeds $\left(5 \mathrm{~cm}^{3} / \mathrm{s}\right)$

\subsection{D CT analysis of specimens of the same bead diameter and same weight percentage with different injection molding speeds}

Different injection molding speeds $(5,20,80 \mathrm{~cm} 3 / \mathrm{s})$ resulted in different bead distributions ranging. As shown in Figure 5, the density of the glass beads is volcanic and tunnel shaped. The darker regions on the images indicate the density increase of the glass beads.

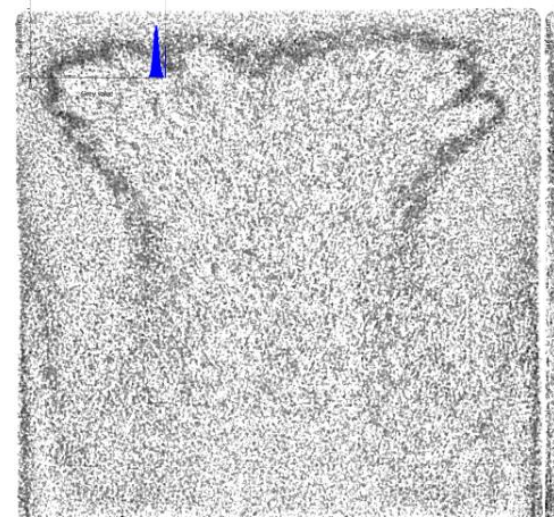

a) 4012505 sample

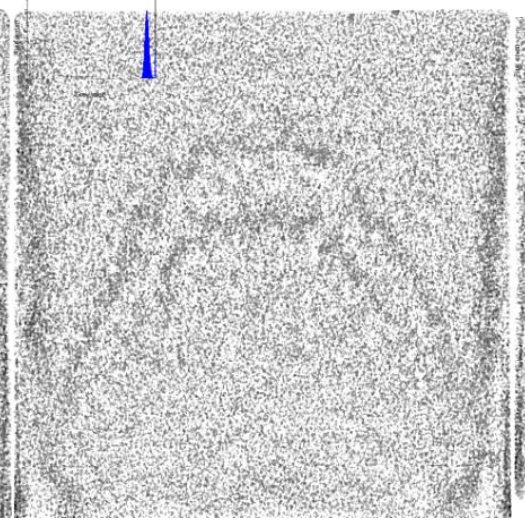

b) 4012520 sample

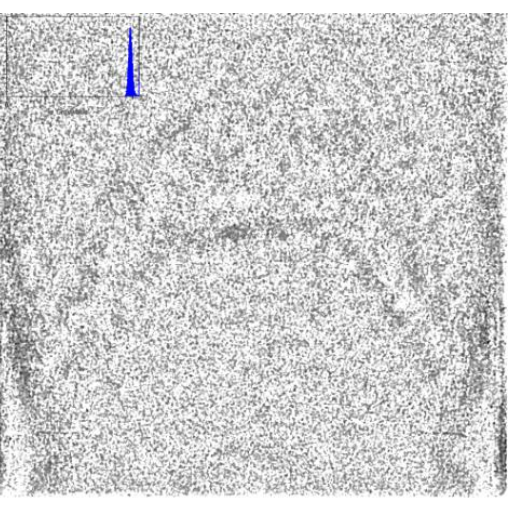

c) 4012580 sample

Figure 5. 2D CT images of PP composite samples containing the same glass bead content (40 wt\%) produced at the different injection molding speeds $(5,20,80 \mathrm{~cm} 3 / \mathrm{s})$

\subsection{D CT analysis of specimens of the same weight percentage with different bead diameter}

In the following studies, we examined whether the size of glass beads affects the distribution of glass beads during injection molding. We found that yes, the glass bead distribution is not uniform. Figure 6 shows the uneven distribution patterns. 


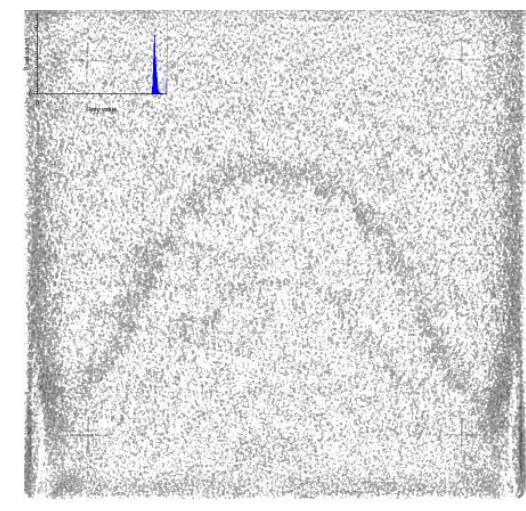

a)40 07580 sample

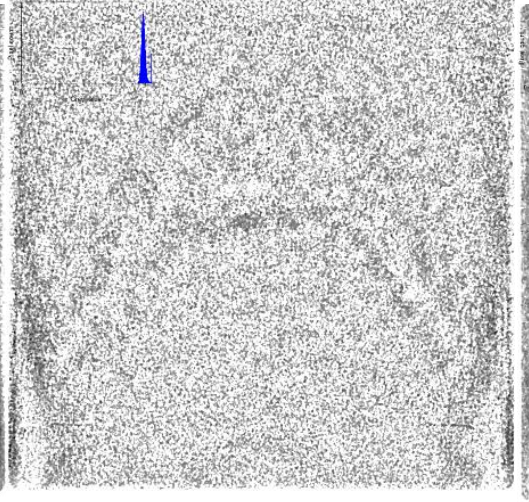

b) 4012580 sample

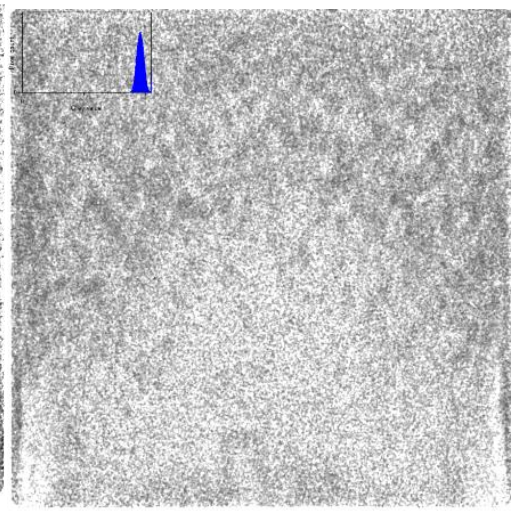

c) 4025080 sample

Figure 6. 2D CT images of PP composite samples containing the same glass bead content (40 wt\%) produced at the same injection molding speeds ( $80 \mathrm{~cm} 3 / \mathrm{s}$ ) and different bead diameters

\subsection{D CT analysis of specimens}

3D CT measurements can be used to determine the location of areas rich and poor in glass beads. This gives an accurate overview of the distribution of the glass beads along the injection molding path. The amount and size distribution of the glass bead were determined at the positions marked x (Figure 7).

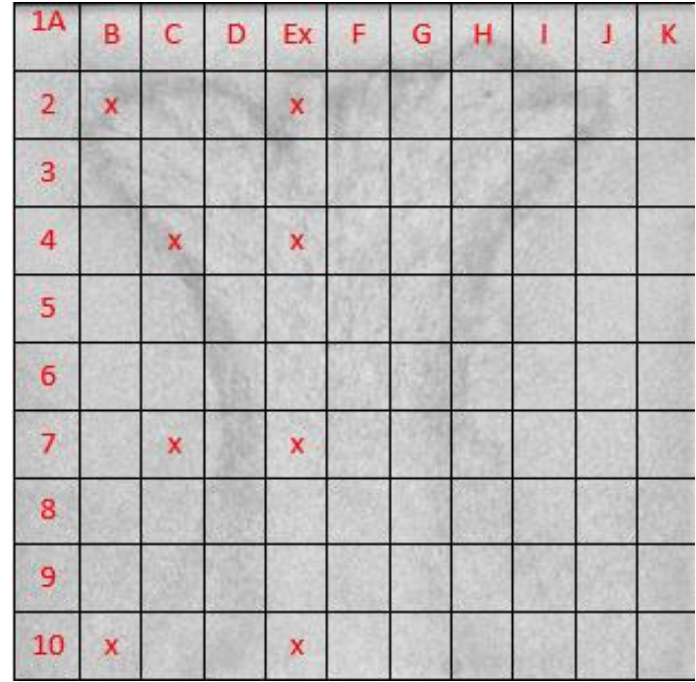

Figure 7. Areas marked with $\mathrm{x}$ in the sample 4007505

The composite sheet has an average glass bead content of 40 weight \%, which corresponds to 18.8 volume \%. The glass bead content is 23,7 volume $\%$ in $2 \mathrm{~B}$ position, and 15 volume $\%$ in $10 \mathrm{E}$ position. Figure 8 shows the distribution of glass bead diameters at positions $2 \mathrm{~B}$ and $10 \mathrm{E}$. 


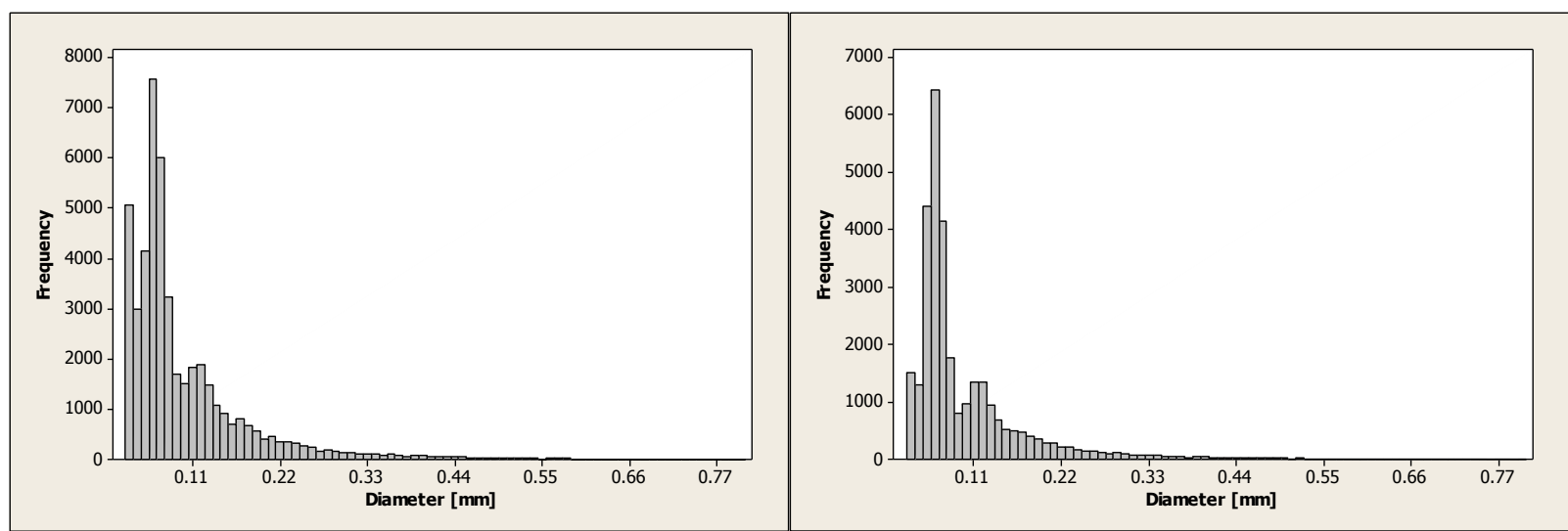

Figure 8. The distribution of glass bead diameters of 4007505 sample - in 2B position, and in $10 E$ position The distribution of glass beads at a test region on a sample plate after 3D reconstruction shows Figure 9. PP composite samples 4007520_07 / 9E after performing the inclusion analysis, the bottom right corner shows the 3D distribution of the glass beads, the top left and the right and the bottom left the $2 \mathrm{D}$ sectional views of the 3D pattern in the $\mathrm{xy}, \mathrm{yz}$, and $\mathrm{xz}$ planes respectively.

The distribution of glass beads is inhomogeneous, with two narrow bands of glass beads clearly visible. It can be seen that by 3D reconstruction the internal structure of the investigated composite can be well mapped.

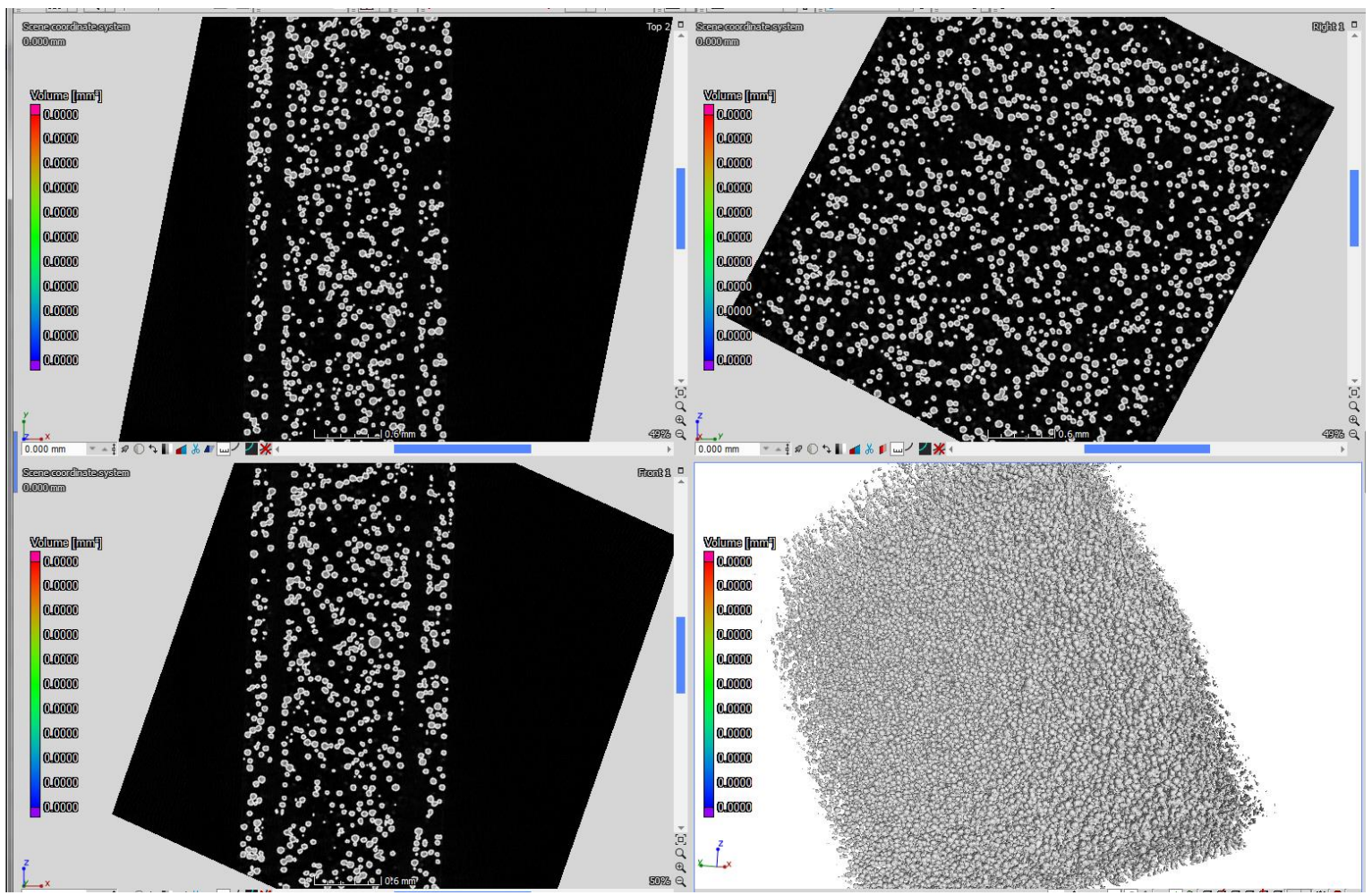

Figure 9. PP composite samples 4007520_07 / 9E after performing the inclusion analysis (the bottom right corner shows the $3 D$ distribution of the glass beads, the top left and the right and the bottom left the $2 D$ sectional views of the $3 D$ pattern in the $x y, y z$, and $x z$ planes respectively) 
International Journal of Engineering and Management Sciences (IJEMS) Vol. 5. (2020). No. 2

DOI: 10.21791/IJEMS.2020.2.23.

\section{Conclusion}

3D CT measurements can be used to determine the location of areas rich and poor in glass beads. This gives an accurate overview of the distribution of the glass beads along the injection molding path. The CT investigation method can be an effective tool for designing filler material (size, volume) and injection molding parameters of particulate composites to ensure uniform filler distribution.

\section{Acknowledgments}

The described work was carried out as part of a project supported by the National Competitiveness and Excellence Program - NVKP_16-1-2016-0038.

\section{References}

[1] Goldstein, J. I. et al. (2018), Scanning Electron Microscopy and X-Ray Microanalysis. Springer International Publishing, 4th ed., Germany.

[2] J. Elek, L.A. Gömze , (2013) Scanning electron microscopic study of glass container degradation in infusion solution, IOP Conference Series: Materials Science and Engineering 47

[3] Carmignato, S., Dewulf W., Leach R. (eds.), (2018), Industrial X-ray Computed Tomography. Springer International Publishing, Germany.

[4] Reinhart, C. (2010), VG Studio MAX: Application examples from science and industry, HighResolution X-ray CT Symposium, Dresden, Germany.

[5] Laurent Babout, (2006) X-Ray Tomography Imaging: a Necessary Tool for Materials Science, Automatyka, 10/3.117-124.

[6] Donald R. Askeland, Pradeep P. Phulé, (2007) The Science and Engineering of Materials, ISBN 81- 315-0321-6 